\title{
Quantum Critical Behavior for a Model Magnet
}

\author{
D. Bitko and T.F. Rosenbaum \\ The James Franck Institute and Department of Physics, The University of Chicago, Chicago, Illinois 60637 \\ G. Aeppli \\ NEC Research Institute, 4 Independence Way, Princeton, New Jersey 08540
}

(Received 18 March 1996)

\begin{abstract}
The classical, thermally driven transition in the dipolar-coupled Ising ferromagnet $\mathrm{LiHoF}_{4}\left(T_{c}=\right.$ $1.53 \mathrm{~K})$ can be converted into a quantum transition driven by a transverse magnetic field $H_{t}$ at $T=0$. The transverse field, applied perpendicular to the Ising axis, introduces channels for quantum relaxation, thereby depressing $T_{c}$. We have determined the phase diagram in the $H_{t}-T$ plane via magnetic susceptibility measurements. The critical exponent, $\gamma=1$, has a mean-field value in both the classical and quantum limits. A solution of the full mean-field Hamiltonian using the known $\mathrm{LiHoF}_{4}$ crystal-field wave functions, including nuclear hyperfine terms, accurately matches experiment. [S0031-9007(96)00753-3]
\end{abstract}

PACS numbers: 75.40.Cx, 05.30.-d, 75.50.Dd

Quantum phase transitions can differ fundamentally from their classical counterparts because of the unparalleled influence of the dynamics on the $T=0$ static critical behavior [1]. In addition, unusual electronic and magnetic behavior can arise at nonzero temperature. Thus includes the peculiar mix of the spin and charge degrees of freedom in transition-metal oxides [2], the apparent "non-Fermi-liquid" behavior of highly correlated $f$-electron compounds [3,4], and the unusual normal-state properties of the high- $T_{c}$ superconducting cuprates [5-8]. The remarkable properties of these systems have been ascribed in each case to the proximity of a $T=0$ quantum critical point.

There remain considerable experimental and theoretical barriers to describing quantum phase transitions with fidelity and precision. In the high-temperature superconductors, for example, the superconductivity masks the direct study of the quantum order-disorder transition. In heavy-fermion materials, characterization of the $T=0$ magnetic instability is complicated by the presence of charge carriers and by substitutional disorder. In spin glasses [9], the combination of frustration and disorder impedes consensus on a correct description of even the thermally driven transition. Moreover, despite their power and elegance, pressure-tuning studies of quantum critical points $[2,3,10]$ cannot approach the exactitude which has become the hallmark of experiments on classical critical phenomena.

High-precision measurements of quantum critical behavior in clean, insulating magnets simply do not exist, even with the great current interest in quantum phase transitions. Therefore, we have carried out such measurements for a model magnet $\mathrm{LiHoF}_{4}$. The key conclusion is that the quantum critical behavior is mean-fieldlike, as predicted by long-standing and elegant theory identifying $(T=0)$ quantum phase transitions in $d$ dimensions with thermal phase transitions in $d+1$ dimen- sions [11]. Furthermore, a mean-field theory using known magnetic parameters quantitatively describes the observed magnetic susceptibility in both the quantum and the thermal regimes.

$\mathrm{LiHoF}_{4}$ in an external field $H_{t}$ is the experimental realization of the simplest quantum spin model, namely, the Ising magnet in a transverse magnetic field. The corresponding Hamiltonian is

$$
H=\sum_{i, j}^{N} J_{i j} \sigma_{i}^{z} \sigma_{j}^{z}-\Gamma \sum_{i}^{N} \sigma_{i}^{x},
$$

where the $\sigma$ 's are Pauli spin matrices, the $J_{i j}$ 's are longitudinal couplings, and $\Gamma$ is a transverse field. Since the commutator $\left[H, \sigma^{z}\right]$ is finite when $\Gamma \neq 0$, zero-point fluctuations are germane at low temperatures. These fluctuations increase with $\Gamma$, which tunes an orderdisorder transition at $T=0$.

In our experiments, the magnetic field $H_{t}$ is applied perpendicular to the easy $(c-)$ axis for the Ho spins. At low temperatures $(T<2 \mathrm{~K})$, the only $\mathrm{Ho}^{3+}$ crystal field state which is appreciably populated is the $\left(H_{t}=0\right)$ ground-state doublet, which can be split in continuous fashion with great precision by the laboratory field $H_{t}$ $[9,12]$. The splitting $\Gamma$ plays the role of the transverse field in Eq. (1), while the doublet plays the role of the spin-1/2 eigenstates. We focus here on the pure system $\mathrm{LiHoF}_{4}$, which is a ferromagnet with a Curie temperature $T_{c}=1.53 \mathrm{~K}$.

The sample, a single crystal of $\mathrm{LiHoF}_{4}$, was ground into a sphere of diameter $4.1 \mathrm{~mm}$ to minimize inhomogeneities in the internal field distribution. It was suspended from the mixing chamber of a helium dilution refrigerator inside the bore of an $80 \mathrm{kOe}$ superconducting magnet, with the field direction oriented along the crystal $a$ axis (within $5^{\circ}$ ), perpendicular to the Ising axis (within $0.5^{\circ}$ ). A trim coil oriented along the Ising direction nulled any unwanted longitudinal field 
component. Heat sinking was achieved via sapphire rods, spring loaded against small flats ground onto the sides of the sample. We measured the complete ac susceptibility, $\chi(f)=\chi^{\prime}(f)+i \chi^{\prime \prime}(f)$, along the Ising axis using a standard gradiometer configuration and a digital lock-in technique [13] with an ac excitation amplitude of $0.075 \mathrm{G}$. $\chi^{\prime}(f)$ in the paramagnet was found to be frequency independent below $1 \mathrm{kHz}$ at all temperatures and transverse fields investigated; the measurements reported here were restricted to $f<0.4 \mathrm{kHz}$.

We plot in Fig. 1 both the real and imaginary parts of the susceptibility as a function of $T$ for zero transverse field. $\chi^{\prime}(T)$ diverges at $T_{c}=1.53 \mathrm{~K}$, below which the Ho spins order ferromagnetically [14,15]. At the identical temperature, there is a sharp increase in $\chi^{\prime \prime}(T)$, most likely due to the motion of domain walls [16]. We use this feature in $\chi^{\prime \prime}$, which occurs at the transition for all transverse fields and temperature studied, as an independent criterion to establish $T_{c}$ (or $H_{t}^{c}$ ). This eliminates $T_{c}\left(\right.$ or $H_{t}^{c}$ ) as a fitting parameter. As a check on this procedure, we have confirmed that the lowest-order nonlinear susceptibility $\chi_{3}\left(T, H_{t}\right)$ exhibits a sharp feature at the identical $T_{c}$ (or $H_{t}^{c}$ ) in both the classical and quantum limits.

Before discussing the critical behavior in the quantum limit, we briefly consider the classical limit. The marginal dimensionality, which separates mean-field from non-mean-field behavior, is $d^{*}=3$ for a dipolar-coupled Ising ferromagnet. As a consequence, in 3D the critical behavior of the system is mean field with logarithmic corrections. For example, the susceptibility should diverge as $t^{-1}|\ln t|^{1 / 3}$, where $t$ is the reduced variable $\left|T-T_{c}\right| / T_{c}$ $[17,18]$. While there is experimental evidence for critical forms with logarithmic corrections in $\mathrm{LiHoF}_{4}$ [18], it is difficult to distinguish experimentally between these forms and simple (mean-field) power laws, particularly

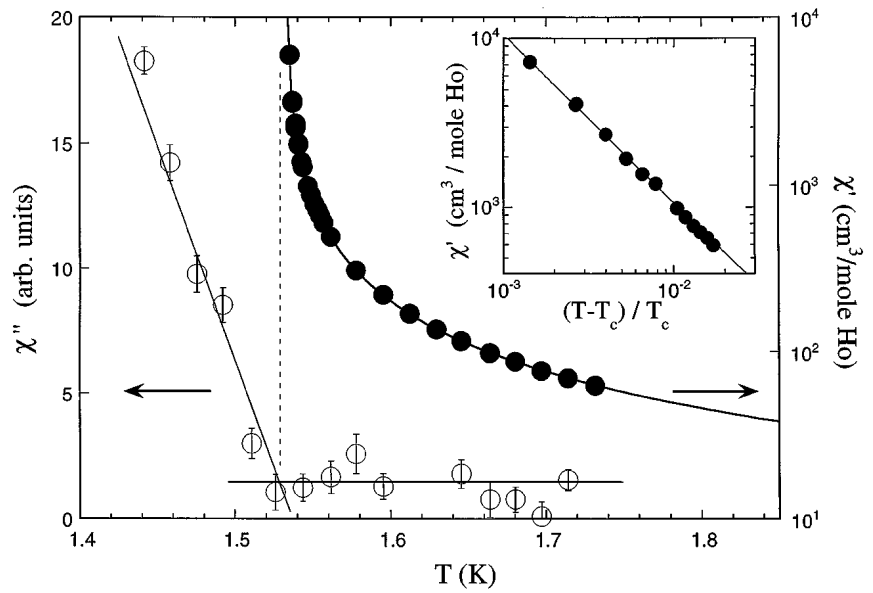

FIG. 1. Divergence of the real part of the magnetic susceptibility (filled circles) and sharp increase in the imaginary part (open circles) at the thermally driven ferromagnetic transition in $\mathrm{LiHoF}_{4}$. Inset: Mean-field critical behavior with $\chi^{\prime} \propto t^{-\gamma}$ and best-fit value $\gamma=1.00 \pm 0.09$ (line). in a susceptibility measurement [15]. We therefore limit ourselves in the Letter to discussing only the leadingorder critical behavior, ignoring any logarithmic corrections which may be present.

The inset of Fig. 1 shows our data for the classical $\left(H_{t}=0\right)$ limit in the critical region. We fix $\chi^{\prime}$ by a simple power law $\chi^{\prime} \propto t^{-\gamma}$, and find a best-fit value $\gamma=$ $1.00 \pm 0.09$. The error bars for this exponent, and the other exponents we measure, are dominated by systematic errors in the demagnetization correction of the data, and not by statistical errors. By comparison, susceptibility measurements by Beauvillain et al. [15] find a best-fit value $\gamma=1.05$.

By crossing the phase boundary varying either temperature or transverse field, we can study the critical behavior of the system throughout the $H_{t}-T$ plane. In the quantum limit, a large field $H_{t}$ is required to traverse the phase boundary. At $T=0.100 \mathrm{~K}$, we find $H_{t}^{c}=49.3 \mathrm{kOe}$. We again fit the susceptibility with a power law of the form $\chi^{\prime} \propto h^{-\gamma}$, where $h=\left|H_{t}-H_{t}^{c}\right| / H_{t}^{c}$ and the critical exponent is independent of the choice of $H_{t}$ or $\Gamma$ for the reduced variable. Beauvillain et al. [15] found the upper limit of the critical region to be $t \sim 10^{-2}$ in the classical regime, while Griffin et al. [18] found it to be $t \sim 10^{-1}$. Although the size of the critical region in the quantum limit may be unrelated, to be conservative we use a critical region of $10^{-3}<h<10^{-2}$ when analyzing the transverse field scan (solid circles in Fig. 2). We find $\gamma=1.07 \pm 0.11$. Approaching the transition in $T$ (at fixed $H_{t}=49.0 \mathrm{kOe}$ ) at nearly the same point in the $H_{t}-T$ plane, we find a distinct upturn in $\chi^{\prime \prime}$ and a divergence of $\chi^{\prime}$ at $T_{c}=0.114 \mathrm{~K}$, with $\gamma=1.01 \pm 0.08$ (open circles in Fig. 2). For $T \leq 0.1 \mathrm{~K}$ our temperature scans are limited to $t=10^{-2}$, we analyze these data assuming a critical region of $10^{-2}<t<10^{-1}$.

We find $\gamma=1$ within error bars at all temperatures studied, down to the lowest temperature probed, $T=$ $0.050 \mathrm{~K}$ or $3 \%$ of $T_{c}\left(H_{t}=0\right)$. Hence, we conclude that the critical behavior at the quantum ferromagnetic transition in $\mathrm{LiHoF}_{4}$ retains its mean-field character. This observation verifies the theoretical prediction [11] that the $T=0$ critical exponents of a $d$-dimensional Ising model in transverse field are equivalent to those of a $(d+1)$ diemensional Ising model in zero transverse field. Studies of the $d=3$ Ising antiferromagnet $\mathrm{MnCl}_{2} \cdot 4 \mathrm{H}_{2} \mathrm{O}$ [19] suggested a crossover to four-dimensional behavior near $T=0$, but did not fully reach the quantum limit.

By repeating the same procedure at other temperatures, we have mapped out the phase diagram shown in Fig. 3. Upon observing mean-field-like critical behavior in both the classical and quantum limits in $\mathrm{LiHoF}_{4}$, it is natural to ask whether the entire phase diagram can be explained in terms of mean-field theory. In fact, in the region $T>0.6 \mathrm{~K}$ the phase diagram has a shape which is well described by the mean-field formula for an ordered $S=$ $\frac{1}{2}$ magnet in transverse field, $\operatorname{coth}\left(\Gamma / 2 k T_{c}\right)=J / \Gamma[20]$. 


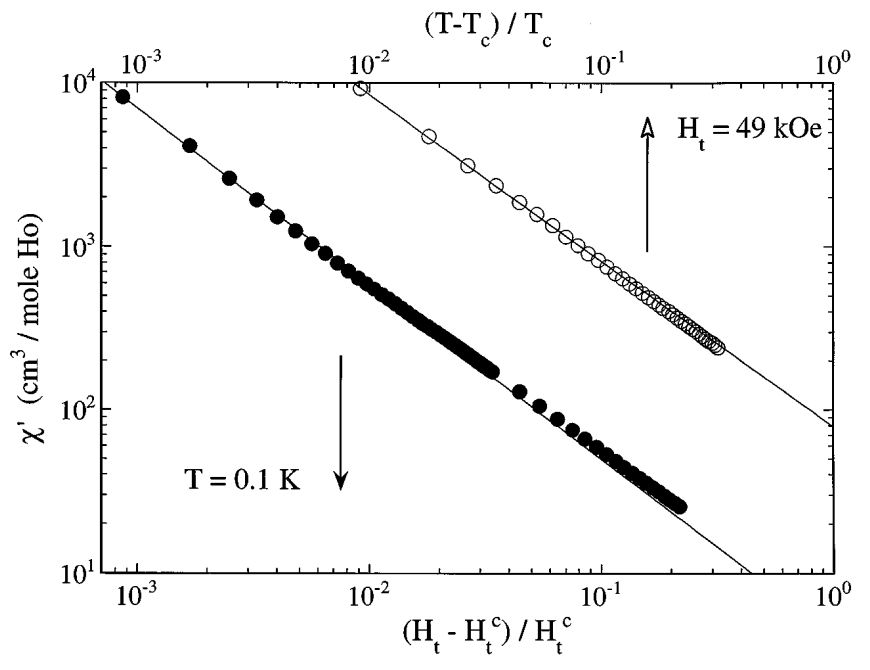

FIG. 2. Mean-field critical behavior of the magnetic susceptibility in the $T \rightarrow 0$ limit as functions of reduced temperature (open circles, $T_{c}=0.114 \mathrm{~K}, H_{t}=49.0 \mathrm{kOe}$ ) and reduced transverse field (filled circles, $H_{t}^{c}=49.3 \mathrm{kOe}, T=0.100 \mathrm{~K}$ ).

Here $J$ is a measure of the interaction between spins, $k$ is Boltzmann's constant, and $\Gamma$ is dependent on the mixing by $H_{t}$ of the ground-state doublet with the excited crystalfield states.

The exact mean-field phase boundary can be calculated by solving the Hamiltonian for a single $\mathrm{Ho}^{3+}$ ion $(J=$ $8, I=\frac{7}{2}$ ) self-consistently:

$$
H=V_{c}-g_{\perp} \mu_{B} H_{t} \hat{J}_{x}+A(\hat{I} \cdot \hat{J})-2 J_{0}\left\langle\hat{J}_{z}\right\rangle \hat{J}_{z},
$$

where $V_{c}$ represents the zero-field crystal-field operator [12], $g_{\perp}$ is the transverse $g$ factor, $A$ is the hyperfine coupling strength, and $J_{0}$ is an averaged spin-spin lon-

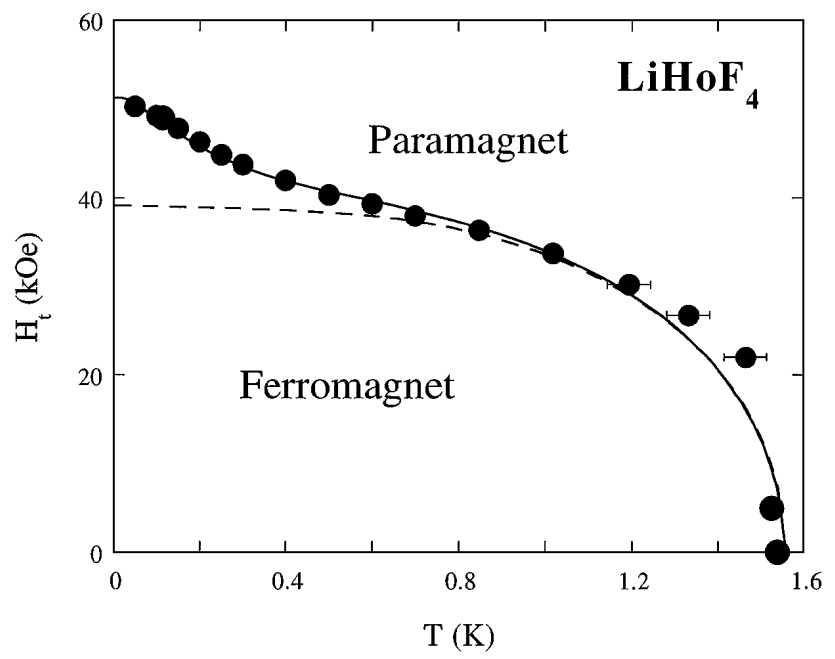

FIG. 3. Experimental phase boundary (filled circles) for the ferromagnetic transition in the transverse field-temperature plane. Dashed line is a mean-field theory including only the electronic spin degrees of freedom; solid line is a full meanfield theory incorporating the nuclear hyperfine interaction [Eq. (2)]. Both theories have the same two fitting parameters. gitudinal coupling constant. The hyperfine term arises from the interaction of the Ho nuclear spins with the electronic states through a core polarization effect [21], and its practical relevance for the magnetic ordering of quantum magnets in effective transverse fields was first noted by Andres for $\mathrm{PrCu}_{2}$ [22]. For $\mathrm{LiHoF}_{4}$, both heat capacity [21] and hyperfine resonance [23] measurements at low $T$ give $A=0.039 \mathrm{~K}=\left(A_{\|}\right) g / g_{\|}$, where $A_{\|}=0.43 \mathrm{~K}$, the Landé $g$-factor $g=1.25$, and the ground-state longitudinal $g$-factor $g_{\|}=13.8$.

A solution for $T_{c}$ as a function of $H_{t}$ is found by fixing $H_{t}$ and then calculating $\left\langle\hat{J}_{z}\right\rangle$ self-consistently, starting at a high temperature and then decreasing $T$ in small steps until a nonzero (spontaneous) magnetization is observed. The hyperfine interaction effectively mixes the nuclear and electronic eigenstates together; therefore, the solution proceeds by diagonalizing Eq. (2) in a $(136 \times$ 136) eigenfunction space ( 17 crystal field states $\times 8$ nuclear states). The solution is shown in Fig. 3 as the solid line, providing an excellent account of the experimental data. We find best fit values $J_{0}=0.0270 \pm 0.0005 \mathrm{~K}$ and $g_{\perp}=0.74 \pm 0.04$. The value $J_{0}=0.0270 \mathrm{~K} \cong$ $2\left\{T_{c}\left(H_{t}=0\right)\right\}\left\{g / g_{\|}\right\}^{2}$. The experimentally determined value of $g_{\perp}$ is remarkably close to the single ion Landé $g$ factor given the large uncertainty in the matrix elements of $J_{x}$ which connect the ground state and excited state crystalfield levels. These matrix elements are calculated from the eigenstates of $V_{c}$ and depend on the measurements which not only contain statistical errors $\geq 25 \%$, but are interpolated from the dilute limit (lightly doped $\mathrm{LiYF}_{4}$ ) [12].

We can illuminate the underlying physics and recover the more conventional mean-field form of the phase diagram by fixing $J_{0}$ and $g_{\perp}$ to their best-fit values and setting $A=0$ in Eq. (2). Solving self-consistently for the magnetization gives the dashed line in Fig 3. At high temperature, $\mathbf{J}$ is the only pertinent quantum number. At low $T$, however, the eigenstates of $\hat{I}$ and $\hat{J}$ are slaved together, and an effective composite spin $(\mathbf{I}+\mathbf{J})$ raises the transverse field scale required to destroy the ferromagnetic state. Hence, it is clear that the upturn in the phase boundary for $T<0.6 \mathrm{~K}$ results directly from the inclusion of the wellknown $\mathrm{Ho}^{3+}$ hyperfine term in the Hamiltonian. Given the spherical symmetry of the hyperfine interaction and the nuclear eigenstates, the hyperfine interaction would not shift the axis of quantization if the electronic crystal-field states also possessed spherical symmetry. In $\mathrm{LiHoF}_{4}$, spherical symmetry is broken by the strongly Ising nature of the crystal-field states and the effect of the hyperfine term is large for $T \leq A_{\|}$.

As a further test that the full mean-field Hamiltonian of Eq. (2) is an accurate description of the physics, we use it to calculate the susceptibility $\chi^{\prime}\left(H_{t}\right)$ of $\mathrm{LiHoF}_{4}$ in the paramagnet. The calculation is performed by adding a small $\left(\sim 10^{-3}\right.$ Oe $)$ longitudinal field $h_{z}$ to the Hamiltonian and solving self-consistently for the magnetization $M_{z}$ with no floating parameters The susceptibil- 
ity $\chi^{\prime}$ is then $M_{z} / h_{z}$, where we have checked explicitly that no higher order terms in $h_{z}$ are present. We plot in Fig. 4 the measured $\chi^{\prime}\left(H_{t}\right)$ at two temperatures, one in the classical regime $(T=1.018 \mathrm{~K})$ and one in the quantum regime where the hyperfine term has a large effect $(T=0.200 \mathrm{~K})$, together with the calculated values of $\chi^{\prime}$. The congruence of experiment and theory shows that a complete mean-field treatment can predict accurately both the functional form and the absolute value of the susceptibility as it falls off in the paramagnet with increasing transverse field.

In summary, we have examined the critical behavior of a 3D Ising ferromagnet in the classical regime by varying temperature, and at the quantum critical point by varying a transverse magnetic field near $T=0$. A full mean-field theory in the $(136 \times 136)$ eigenfunction space of the 17 crystal-field and 8 nuclear states of the $\mathrm{Ho}^{3+}$ ion accounts quantitatively for the critical behavior of $\chi^{\prime}\left(T, H_{t}\right)$, the shape of the phase boundary between paramagnet and ferromagnet, and the temperature and transverse field evolution of the susceptibility well into the paramagnet. With a quantitative understanding of the clean, insulating, quantum ferromagnet, it should now be possible to address the effects of spin disorder [24], and perhaps even charge carriers, on quantum critical phenomena.

We have benefited greatly from discussions with R. Wickham. The work at the University of Chicago was supported primarily by the MRSEC Program of the National Science Foundation under Award No. DMR-9400379.

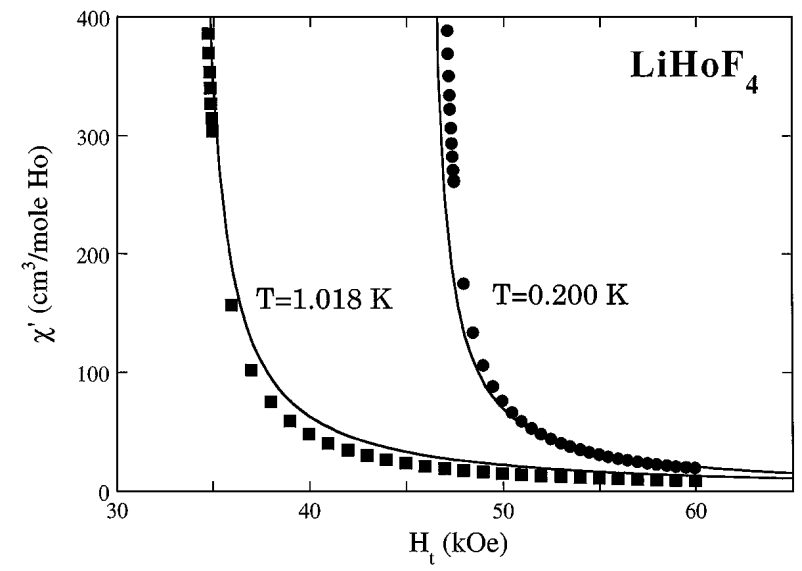

FIG. 4. Transverse field dependence of the susceptibility in the paramagnet for two temperatures. Solid line is a first principles calculation with parameters fixed by the fit to the phase boundary of Fig. 3 .
[1] J. A. Hertz, Phys. Rev. B 14, 1165 (1976); M. A. Contentino, ibid. 47, 11587 (1993); A. J. Millis, ibid. 48, 7183 (1993).

[2] S. A. Carter et al., Phys. Rev. Lett. 67, 3440 (1991); Phys. Rev. B 48, 16841 (1993).

[3] H. v. Löhneysen et al., Phys. Rev. Lett. 72, 3262 (1994); B. Bogenberger and H. v. Löhneysen, ibid. 74, 1016 (1995).

[4] For a review, see M. B. Maple et al., J. Low. Temp. Phys. 99, 223 (1995).

[5] S. Chakravarty, B. I. Halperin, and D. R. Nelson, Phys. Rev. B 39, 2344 (1989).

[6] S. Sachdev and J. Ye, Phys. Rev. Lett. 69, 2411 (1992); A. Chubukov and S. Sachdev, ibid. 71, 169 (1993); A. Sokol and D. Pines, ibid. 71, 2813 (1993); A. W. Sandvik and D. J. Scalapino, ibid. 72, 2777 (1994); A. W. Sandvik and M. Vekic, ibid. 74, 1226 (1995).

[7] B. Keimer et al., Phys. Rev. Lett. 67, 1930 (1991); S. Hayden et al., ibid. 66, 821 (1991).

[8] T. Imai et al., Phys. Rev. Lett. 70, 1002 (1993); 71, 1254 (1993).

[9] W. Wu et al., Phys. Rev. Lett. 67, 2076 (1991); 71, 1919 (1993).

[10] D. B. McWhan, C. Vettier, R. Youngblood, and G. Shirane, Phys. Rev. B 20, 4612 (1979).

[11] M. Suzuki, Prog. Theor. Phys. 56, 1454 (1976); J. Oitmaa and G. J. Coombs, J. Phys. C 14, 143 (1981).

[12] P. E. Hansen, T. Johansson, and R. Nevald, Phys. Rev. B 12, 5315 (1975).

[13] N. O. Birge and S. R. Nagel, Rev. Sci. Instrum. 58, 1164 (1987); P. K. Dixon and L. Wu, ibid. 60, 3329 (1989).

[14] A. H. Cooke, D. A. Jones, J. F. A. Silva, and M. R. Wells, J. Phys. C 8, 4083 (1975).

[15] P. Beauvillain et al., Phys. Rev. B 18, 3360 (1978).

[16] J. Kötzler, M. Grahl, I. Sessler, and J. Ferré, Phys. Rev. Lett. 64, 2446 (1990).

[17] A. Aharony and B. I. Halperin, Phys. Rev. Lett. 35, 1308 (1975); E. Brezin and J. Zinn-Justin, Phys. Rev. B 13, 251 (1976); G. Ahlers, H. Kornblitt, and H.J. Guggenheim, Phys. Rev. Lett. 34, 1227 (1975); J. Als-Nielsen, ibid. 37, 1161 (1976).

[18] J. A. Griffin, M. Huster, and R. J. Folweiler, Phys. Rev. B 22, 4370 (1980).

[19] W. A.C. Erkelens et al., Europhys. Lett. 1, 37 (1986); J. J. M. Steijger et al., J. Magn. Magn. Mater. 31-34, 1091 (1983).

[20] R. W. Youngblood, G. Aeppli, J. D. Axe, and J. A. Griffin, Phys. Rev. Lett. 49, 1724 (1982).

[21] G. Mennenga, L. J. deJongh, and W. J. Huiskamp, J. Magn. Magn. Mater. 44, 59 (1984).

[22] K. Andres, Phys. Rev. B 7, 4295 (1973).

[23] J. Magarinõ, J. Tuchendler, P. Beauvillain, and I. Laursen, Phys. Rev. B 21, 18 (1980).

[24] D. H. Reich et al., Phys. Rev. B 42, 4631 (1990). 